Supporting Information

\title{
Early Stage Interplay of Microphase Separation and Crystallization in Crystalline-Coil Poly(L-Lactic Acid)-block-Polystyrene Thin Films
}

Jun Fu, Bin Luan, Caiyuan Pan, Binyao Li, and Yanchun Han*

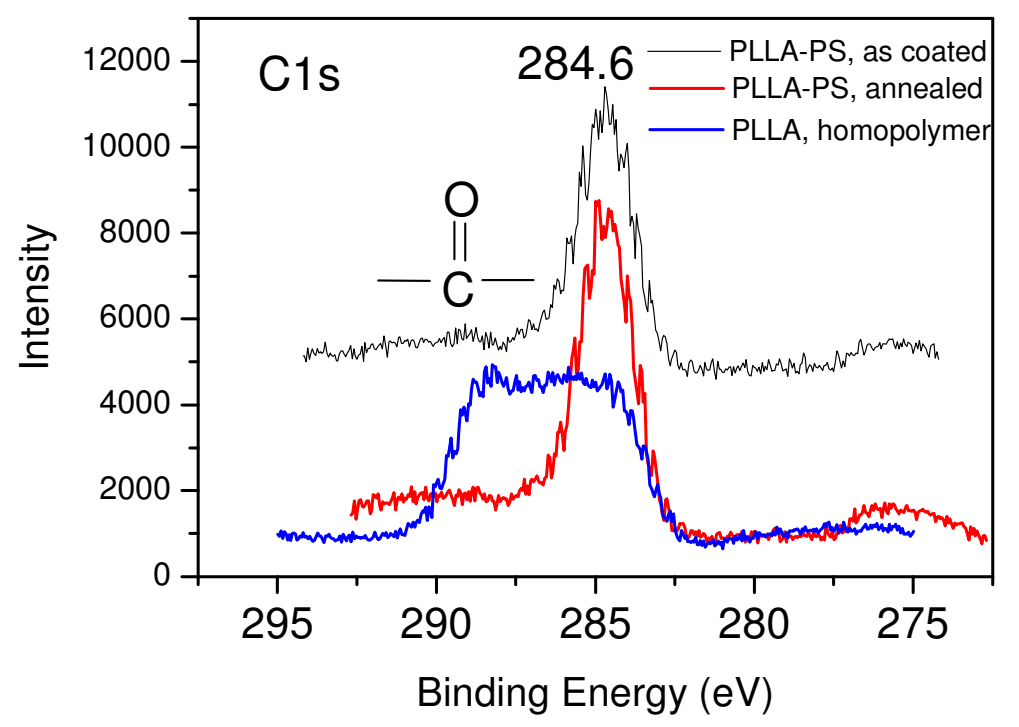

Figure S1. XPS spectra for the PLLA-b-PS thin film before (dark) and after (red) annealing. The XPS spectrum for the PLLA homopolymer (blue) is also included for comparison. The take-off angle is $90^{\circ}$, indicating a penetration depth of less than 10 $\mathrm{nm}$. Slight shift in the peak at higher binding energy side may be owed to the linkage to the PS block. The very weak signal for the carbonyl group for the unannealed PLLA-b-PS film is resulted from the very low mole fraction of the carbonyl group in the block copolymer. Besides, the high surface energy PLLA blocks prefer to segregate to the internal of the films during spin coating, further decreasing the fraction of the carbonyl group at the surface. 

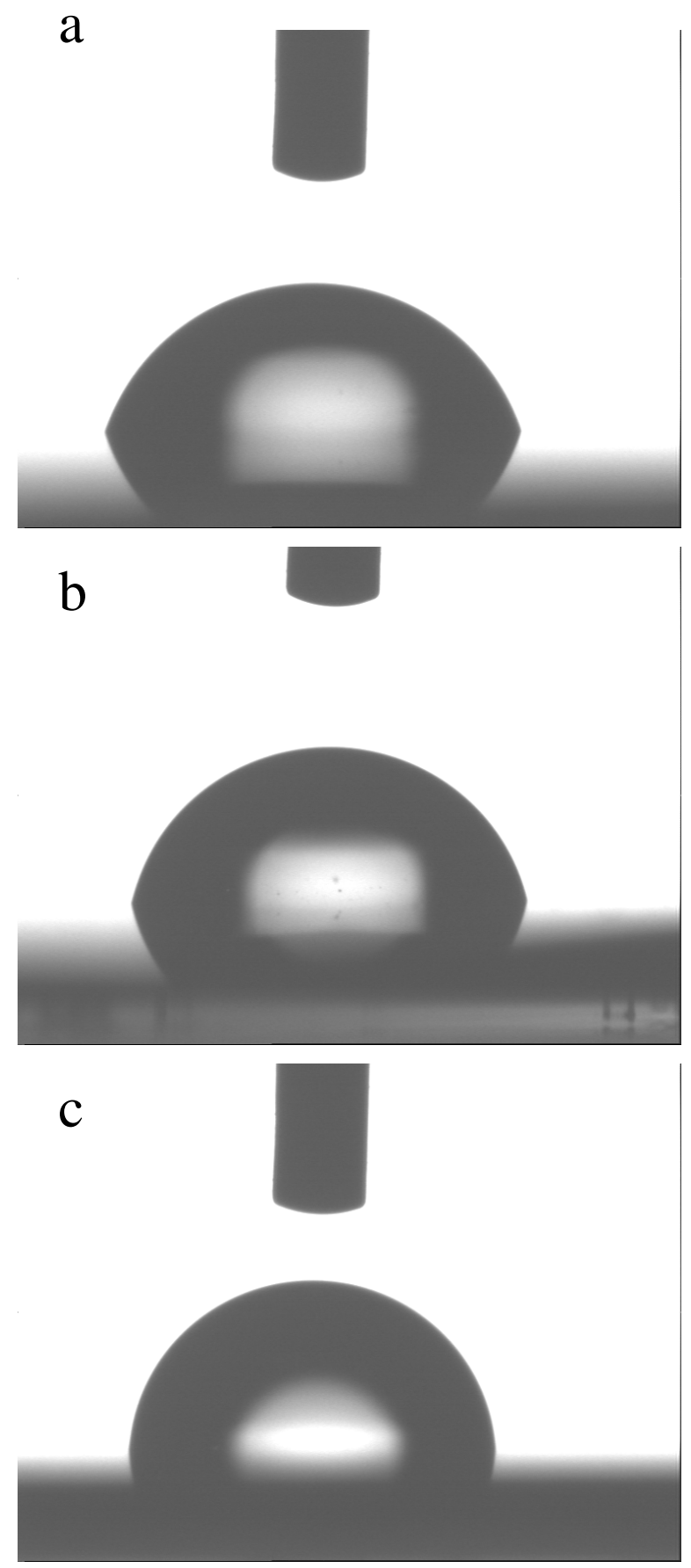

Figure S2. The contact angles of (a) PLLA homopolymer, $\sim 73^{\circ}$, (b) spin-coated PLLA-b-PS thin films, $\sim 80^{\circ}$, and (c) PLLA-b-PS films after annealing at $180{ }^{\circ} \mathrm{C}$, $\sim 91^{\circ}$. The increase in the contact angle after annealing indicates the segregation of PS (contact angle $=91^{\circ}$ ) to the surface . 